\title{
La educación para la igualdad, la diferencia y la enseñanza de la historia de las mujeres: reflexiones y desafíos
}

\author{
Jesús Marolla Gajardo ${ }^{1}$ \\ Recibido: 10 de abril de 2015 - Aceptado: 20 de mayo de 2015
}

\begin{abstract}
Resumen
Actualmente los estudios de género, inclusión, diversidad e igualdad son temáticas cruciales que hay que considerar si queremos que la formación estudiantil en historia y ciencias sociales tenga por finalidad la formación de una sociedad más justa, igualitaria y respetuosa de las diferencias. Las reflexiones que se recogen a continuación son parte de los esfuerzos que se realizan desde distintos sectores por una enseñanza más inclusiva. La educación para la igualdad y la diferencia presenta aspectos teóricos pensados en prácticas y escuelas que se consideren heterogéneas y diversas. La coeducación viene a presentar criterios sobre un tipo de organización escolar que considere el trabajo en conjunto con el alumnado y la enseñanza para la vida democrática como aspectos esenciales en la formación. Ambas propuestas son esenciales para democratizar la enseñanza de la historia y las ciencias sociales e incluir a las mujeres en la historia bajo las mismas categorías que han ostentado los hombres.
\end{abstract}

Palabras clave: historia de las mujeres, didáctica, enseñanza, diversidad, igualdad.

\section{Education for equality, difference and teaching of women's history: challenges and reflections}

\begin{abstract}
Today, studies about gender, inclusion, diversity and equality are crucial topics that must be considered if we want the student training in history and social studies is dedicated to the formation of a more egalitarian society that respects differences. Reflections gathered are part of the efforts from various sectors
\end{abstract}

Chileno. Doctorando en Educación en Didáctica de las Ciencias Sociales. Departament de Didàctica de la Llengua i la Literatura, i de les Ciències Socials, Universitat Autònoma de Barcelona, España. Este trabajo es parte de mi proyecto de investigación titulado "Ellas sí tienen historia: Representaciones sociales y prácticas del profesorado y alumnado sobre la enseñanza de la historia de las mujeres". E-mail: jesusmarolla@gmail.com 
seeking a more inclusive education. Education for equality and difference presents theoretical and practical aspects designed in schools considered as heterogeneous and diverse. Coeducation integrates a school organization with jointly work with students and the teaching for democratic life as essential aspects in training. Both proposals are essential to democratize the teaching of history and social sciences and include women in history under the same categories that men.

Keywords: Women's history, teaching, education, diversity, equality.

\section{Educação para a igualdade, a diferença e ensino da história de mulheres: reflexões e desafios}

\section{Resumo}

Atualmente os estudos de gênero, inclusão, diversidade e igualdade são temas cruciais que devem ser considerados se queremos que a formação estudantil em história e nas ciências sociais tenha por objeto a formação de uma sociedade mais justa, igualitária, respeitosa das diferenças. As reflexões que se recolhem a continuação são parte dos esforços que se realizam desde diversos setores para uma educação mais inclusiva. A educação para a igualdade e a diferença apresenta aspectos teóricos pensadas em práticas e escolas que se considerem heterogêneas e diversas. A coeducação apresenta critérios sobre um tipo de organização escolar que considere o trabalho em conjunto com os alunos e o ensino para a vida democrática como aspectos essenciais na formação. Ambas as propostas são essenciais para democratizar o ensino da história e das ciências sociais e incluir as mulheres na história sobe as mesmas categorias que têm assumido os homens.

Palavras-chave: história das mulheres, didática, ensino, diversidade, igualdade.

\section{La diversidad en las escuelas y las aulas}

"No es la misma templanza la de la mujer que la del hombre, ni la misma fortaleza, como creía Sócrates, sino que la del hombre es una fortaleza para mandar, la de la mujer para servir, y lo mismo las demás virtudes"

(Aristóteles, 2006)

Actualmente asistimos a la presencia de una escuela con mayor presencia de diversidades. Principalmente diversidades que nacen desde la clase, la etnia y los géneros. No se puede considerar en los tiempos actuales a las escuelas como centros homogéneos, sino que como plantean Bickmore (1999, 2002, 
2008), C. Banks y J. Banks (2012) y Harris (1996) dentro de la globalización se debe considerar y trabajar desde los contextos particulares en reconocimiento a las diversidades existentes. Lo particular, lo local y la diferencia deben ser los conceptos que guíen los procesos educativos.

De acuerdo a lo que plantean Aubert et al. (2010), Agacinski (1998), Connell (1993), Fernández Valencia $(2004,2006)$ y Scott (2008), existe consenso en cuanto a que el género es una construcción social, donde la cultura, y no la biología, ha puesto a las mujeres durante mucho tiempo y en diversas sociedades en una situación de discriminación y dependencia social. No obstante y de acuerdo a lo que plantea Vega (2002), aún en las escuelas y las aulas se presencian comportamientos, relaciones y una enseñanza basada en los prejuicios de género.

Siguiendo a Vega (2002), es innegable que en las últimas décadas, las sociedades occidentales han experimentado cambios a favor de la igualdad entre hombres y mujeres. Cabe manifestar la pregunta: ¿la igualdad se ha conseguido? ¿Podemos hablar de un mundo igual para hombres y mujeres?

Vega (2002) plantea que en la sociedad existe un largo camino por recorrer para lograr la igualdad de género. En educación el camino que se plantea es aún más largo. En teoría la escuela es impulsora y generadora de cambios sociales; en la práctica, en demasiadas ocasiones las aulas y centros actúan perpetuando las pautas y estereotipos culturales y de género que existen en la sociedad.

Si queremos trabajar en las escuelas desde la igualdad y diversidad, considerando la historia de las mujeres en los mismos niveles en que trabajamos con la historia de los hombres, como plantea Subirats (2001), es necesario romper con las barreras de género y enfocarse en la democratización de los espacios escolares. La vía democrática es el camino efectivo para superar las desigualdades e injusticias que ocurren con la mujer y otros colectivos marginados (Casas, 1999). Como sugieren Oesterreich (2002) y Subirats (2001), romper con las barreras de género es un proceso largo, por ello se debe trabajar en función de promover un marco de justicia social a fin de que todos y todas tengan las mismas posibilidades de acceso al conocimiento. De esta forma los y las partícipes del proceso educativo se identificarán con las materias que se trabajan.

Debido a que la escuela, como plantean Hubbard (2013), Smith (1996), Stone (1996) y Vázquez (2003), está organizada desde el patriarcado, no se recogen temáticas sobre la desigualdad histórica de las mujeres, el androcentrismo en la sociedad, la enseñanza y los centros y la fuerte carga y presencia de los estereotipos sexuales que no solo discriminan sino que también bus- 
can universalizar los valores masculinos. En este sentido, Bonilla y Martínez (1992) concuerdan en afirmar que la escuela juega un papel crucial en la creación y perpetuación de desigualdades para sus alumnos y alumnas, por lo que plantean que:

"Aunque se defiende la igualdad, se practica la diferencia. En el caso del alumnado, esto se manifiesta de varias formas. Por un lado, se observa una sobrevaloración de los propios atributos de género y una devaluación de los asignados socialmente al sexo opuesto, y, por otro, es manifiesta la configuración de grupos por el sexo, siendo el rechazo de la pertenencia de chicas a los grupos masculinos una de las razones que, con mayor frecuencia, provocan conflictos entre ambos sexos" (Bonilla y Martínez, 1992: 69).

Aguirre Sáez de Egilaz (2002) y Applebaum (2004) argumentan que no se puede proponer la igualdad sin de-construir los estereotipos de género en las aulas y la enseñanza de la historia. La escuela, al propugnar un marco de igualdad, lo que realmente está haciendo es fomentar un modelo de escuela masculino. Lo que realmente se está fomentando es la jerarquización de los roles de género, ya que sobrevaloran los atributos que conforman la masculinidad, en cambio, infravaloran los que configuran la feminidad.

Vázquez (2003) argumenta que debido a que la escuela en su generalidad es de tipo mixto provoca que sea percibida como institución de aspecto neutral que no origina discriminación por razón de sexo. No obstante, la creación de la escuela mixta no atiende una reflexión sobre las exigencias de una educación que se inicia desde los marcos de la igualdad. No se reflexiona sobre las diferencias que se manifiestan entre niños y niñas, ya que las chicas se deben incorporar a las normas, valores y comportamientos propios de la cultura dominante.

Vázquez (2003) comenta algunas características sobre la escuela mixta que describen la problemática enunciada:

"-Perspectivas androcéntricas como definidoras de un pseudocurrículo escolar.

-Proyección sexista en materiales didácticos y educativos.

-Existencia de un currículo oculto marcadamente sexista.

-Atribución de privacidad -sobre todo en el ámbito doméstico-como orientación fundamental de la propuesta educativa femenina.

-Uso habitual del lenguaje en función de masculino genérico omnicomprensivo que funciona como un factor de macro-identificación para el alumnado varón" (Vázquez, 2003). 
En cuanto a la enseñanza de la historia, la escuela se encarga de reproducir estereotipos y patrones de género en vez de trabajar por su cambio (Benavente y Núñez, 1992; Bonilla \& Martínez, 1992; Fernández, 2006; Tomé y Rambla, 2001). Si en las aulas solo incorporan conocimientos, valores y experiencias de los grupos dominantes, se margina automáticamente a aquellos y aquellas estudiantes que son parte de las minorías raciales, culturales, lingüísticas, religiosas y sexuales (Banks, 2004, p. 13; Banks y Banks, 2012). Para Álvarez de Zayas y Palomo Alemán (2002) es fundamental que, desde el plano educativo, las y los alumnos se den cuenta de que todos y todas hacemos nuestra historia. Sean historias individuales, privadas, familiares, de hombres y mujeres comunes, siempre son historias.

García y Roset (1992) plantean que si se quieren conseguir cambios efectivos en las relaciones que se han dado en las aulas y en la enseñanza de la historia es necesario considerar la historia de las mujeres en igualdad a la historia de los hombres. No obstante, no basta con enseñar desde la historia de las mujeres, sino que es imperioso cambiar los enfoques con que se han enseñado la historia y las ciencias sociales.

En las escuelas y en la enseñanza de la historia y las ciencias sociales, como afirman García y Roset (1992) y Dam y Rijkschroeff (1996), se crean pautas y valores relacionados con los hombres y las mujeres. Por ejemplo, la escasa atención otorgada a la participación femenina en espacios laborales, genera una visión subordinada de las mujeres en contraste a la alta atención otorgada a las guerras, la política y la economía. De esta manera la escuela y la enseñanza de las ciencias sociales van formando estereotipos y roles culturales.

El cambio de enfoques en la enseñanza debe considerar aspectos como el respeto, la democracia y la diversidad. En este contexto, Banks et al. (2004a; 2005) postulan una serie de principios y conceptos enfocados en la transformación de la enseñanza de la historia, la democracia y la diversidad:

\section{"Principles}

Diversity, Unity, Global Interconnectedness, and Human Rights

1.- Students should learn about the complex relationships between unity and diversity in their local communities, the nation, and the world.

2.- Students should learn about the ways in which people in their community, nation, and region are increasingly interdependent with other people around the world and are connected to the economic, political, cultural, environmental, and technological changes taking place across the planet. 
3.- The teaching of human rights should underpin citizenship education courses and programs in multicultural nation states.

Section II Experience and participation

1. Students should be taught knowledge about democracy and democratic institutions and provided opportunities to practice democracy.

Concepts

1.Democracy.

2.Diversity.

3.Globalization.

4.Sustainable development.

5.Empire, imperialism, power.

6.Prejudice, discrimination, racism.

7.Migration.

8.Identity /diversity.

9.Multiple perspectives.

10.Patriotism and cosmopolitanism." (Banks et al., 2005: 5).

A partir del estudio de Banks, se puede afirmar que es imprescindible que dentro de la historia, la geografía y las ciencias sociales se incluya la historia de las mujeres en igualdad y respetando las diferencias con la historia de los hombres.

\section{El modelo coeducativo}

Dentro de los esfuerzos que han existido desde diversos círculos que trabajan la enseñanza de la igualdad desde la diferencia, y que argumenta que para incluir la historia de las mujeres deben cambiar los enfoques de enseñanza, se ha planteado la instalación del modelo coeducativo. Tal modelo es una herramienta efectiva que se preocupa de que la enseñanza no recoja los prejuicios y estereotipos de género. Este modelo pretende eliminar las jerarquías entre personas y en el conocimiento que se entrega a los y las alumnas.

El sistema de coeducación toma las diferencias entre diversos grupos sexuales y los incorpora dentro de la diversidad que debe existir en las escuelas (Banks, 2006a; Vázquez, 2003; Torney-Purta, et. al., 2001). No se trata de homogeneizar, sino de respetar las diferencias en marcos de igualdad (Aubert et al., 2010; Stone, 1996). Es por esto que Aubert et al. (2010) y Tomé y Rambla (2001) plantean que una de las finalidades de la 
coeducación no es solo superar los arquetipos femeninos impuestos, sino también la superación de los arquetipos y estereotipos masculinos.

Vázquez (2003) define las principales características del modelo de coeducación en:

"-Descubrir, potenciar y desarrollar capacidades personales sin dejarse mediatizar por ninguna limitación de carácter sexista.

-Desarrollar la propia autonomía e identidad personal con independencia de la variable género.

-Desarrollar la capacidad de sentir y expresar los propios sentimientos en las actividades cotidianas.

-Potenciar el valor de la afectividad, de la emoción y de la ternura.

-Descubrir en las experiencias diarias la igualdad de posibilidades y de aportaciones de hombres y mujeres.

- Respetar y valorar las características de las personas sin dejarse influir por actitudes discriminatorias en relación con el sexo o con cualquier otro rasgo diferenciador.

-Integrar el hecho de la diversidad como un valor y una riqueza.

-Experimentar el valor de la complementariedad.

-Participar de modo igualitario en la realización de tareas, rechazando la división del trabajo en función del sexo.

- Colaborar en la construcción de una sociedad basada en el respeto de la individualidad y singularidad de las personas.

- Realizar una análisis crítico de las manifestaciones sexistas que se producen en el lenguaje o en los mensajes que transmiten los medios de comunicación" (Vázquez, 2003).

McIntosh (2005) plantea que para la creación de un ambiente coeducativo, tanto en los centros como en el contenido que se trabaja, es necesario dotar de protagonismo a los y las estudiantes. Tal protagonismo se debe ver reflejado tanto en la construcción del currículo que se enseñará como así también en las decisiones que se lleven a cabo en las aulas y centros (Apple, 2012).

McIntosh (2005) plantea una serie de preguntas sobre la implementación del modelo coeducativo:

"Discuss questions like:

1. What are we teaching?

2. What messages does our teaching deliver with regard to gender, race, class, culture, region, nation, and the world? 
3. What outcomes do we want of education?

4. What Works for students' and our own growth and development?

5. Can we give our students a more complete picture than we were given ourselves, of the self and the world in their many dimensions?

6 . How can we make school climate, teaching methods, and curricula more gender-fair, multicultural, and global?" (Mclntosh, 2005, p. 31).

\subsection{Las prácticas del profesorado en el modelo coeducativo}

Dentro de los lineamientos que definen el modelo educativo el profesorado es fundamental en la guía del proceso de enseñanza y aprendizaje. Aubert et al. (2010) comentan la existencia de tres modelos con los cuales podría caracterizarse la práctica de los y las profesoras: modelo disciplinar, modelo mediador y modelo comunitario (Aubert et al., 2010).

Por un lado, el modelo disciplinar se basa fundamentalmente en la autoridad del profesorado y la aplicación de las normativas diseñadas en la institución escolar. Se inicia desde la preconcepción de que el alumnado tiende al mal comportamiento y a infringir las normas, por lo cual los y las docentes actúan como vigilantes y ejecutores de sanciones. Generalmente, la vigilancia y las sanciones se acrecientan en aquellos grupos marginados tradicionalmente, sean mujeres, homosexuales, etnias, etc. Lo que en definitiva contribuye a generar desigualdades, jerarquías y prejuicios en torno a tales colectivos (Aubert et al., 2010).

El modelo mediador aparece en el momento en que existen conflictos, no como una prevención de ellos. Si bien se experimentan mejoras en las relaciones sociales con la comunidad estudiantil, se demanda constantemente la asesoría de expertos para mediar entre los conflictos y ofrecer soluciones (Aubert, et al., 2010). La interacción en este modelo se produce a través del diálogo profesionalizado, no obstante, tales relaciones pueden provocar que se vuelva a caer en etiquetas.

Helen Cowie (1996) afirma que en el modelo mediador se destacan mejoras como la colaboración y el apoyo. A través de técnicas de ayuda, resolución de conflictos y counselling, se integran a los y las alumnas para la resolución de los conflictos. Se fomenta de esta forma la escucha activa, la empatía y la resolución de problemas. Así se logra una reducción en los estereotipos y los comportamientos discriminatorios (Cowie, 1996, citado por Aubert et al., 2010).

Por último, el modelo comunitario consiste en el fomento a la participación de la comunidad en un clima de igualdad, intercambio y desarrollo de valores democráticos 
Como plantean Aubert y otros (2010), la participación de toda la comunidad en este modelo implica que a través del diálogo y la igualdad se descubran las causas, los orígenes de los conflictos y sus posibles soluciones, acercándose de gran forma al modelo coeducativo. Se trata de crear un clima de colaboración y de reconocimiento mutuo, en donde las personas no se sientan juzgadas a priori y en el que las normas, el funcionamiento del centro y la forma de resolver los conflictos sea decidida conjuntamente por todos los participantes. De esta manera, tanto el profesorado como el alumnado participan en la creación de normas inclusivas para toda la comunidad. Esto contribuye a generar un clima para que todos y todas tengan las mismas oportunidades para expresar su voz y encontrar soluciones a las problemáticas en un clima de igualdad y respeto por los derechos.

\section{Conclusiones: Enseñar la historia de las mujeres desde la igualdad y la diferencia}

Autores como Cummins (2001), Hernández y Metzger (1996) y Deusdad (2010) plantean que la enseñanza desde el multiculturalismo, el género y en concreto la historia de las mujeres debe representar un cambio en las actitudes de los y las alumnas. Para los y las autoras trabajar desde tales temáticas debe propiciar que el alumnado aprenda a desenvolverse en la sociedad actual. Para que el verdadero cambio a nivel educativo se produzca, es necesario incentivar a los y las estudiantes a que se empoderen y comprometan con cambios tanto dentro del aula como fuera. Como dicen Castles (2004) y Pugh y García (1996), los y las alumnas deben saber respetar y reconocer a aquellos grupos marginados tradicionalmente desde una mirada de igualdad.

En Subirats y Brullet (1988), al trabajar con la historia de las mujeres de forma aislada, la feminidad adquirirá pautas de comportamiento que la posicionan en una escala jerárquica menor en comparación a los hombres protagonistas de la historia. Como plantea Aubert et al. (2010), apenas hay mujeres referentes en la historia, así como no parecen existir mujeres filósofas, educadoras o científicas. De esta forma se va creando el estereotipo relacionado a que las mujeres tienen menores capacidades intelectuales que los hombres.

Thorton (2005) señala que para lograr visibilizar a las mujeres y otros actores es necesario problematizar el contenido y posicionar la crítica como conductor del aprendizaje. Por ello el trabajo desde la creación de marcos conceptuales y el rescate de la historia de las mujeres desde una perspectiva no androcéntrica es una manera efectiva de avanzar en la democratización de la sociedad. De la misma manera, McIntosh (2005) argumenta que dentro 
de los estudios sociales y la enseñanza de la historia existe un predominio de las visiones y protagonismo masculinos. La autora plantea que se debe trabajar y rescatar a las mujeres y su historia a fin de que se comiencen a cambiar las pautas y estereotipos sociales.

Barton y Levstik (2004) y Banks (2006b) plantean, como se ha señalado hasta ahora, que la enseñanza de la historia puede generar estereotipos y falsas impresiones en el alumnado. No obstante, un cambio de enfoques en la enseñanza de la historia y las ciencias sociales que posicione a las mujeres en igualdad con los hombres puede contribuir a generar cambios a favor de la disminución de la desigualdad, la discriminación y los estereotipos de género presentes en la sociedad. De esta forma, las acciones de los y las estudiantes destacarán por manifestarse como una ciudadanía comprometida con la construcción de un mundo más justo que respete las diferencias.

Blanco (2004), de la misma manera, afirma que desde los estudios sociales y la enseñanza de la historia se puede contribuir a la justicia social y la igualdad con respeto a las diferencias. Tyson y Hinton-Johnson (2003) y Woyshner (2002) afirman que la sociedad actual no puede seguir reproduciendo la opresión a las mujeres y a las distintas colectividades y grupos marginados tradicionalmente.

Trabajar las temáticas relacionadas con la historia de las mujeres desde la perspectiva de la educación para la igualdad y la diferencia, ofrece diversas posibilidades educativas aplicables a los centros y aulas. Aubert et al. (2010) plantean que si la lucha es por una educación igualitaria, tanto en los establecimientos como en los contenidos que se trabajan, necesariamente las reformas deben pasar por el respeto hacia las diferencias. El concepto de diferencia desde el plano educativo hace alusión al reconocimiento de que existen diversidades entre los géneros, vistas desde un plano de igualdad. Como argumentan Aubert et al. (2010), "cuando en nombre de la igualdad no se tiene en cuenta la diferencia, se impone un modelo homogéneo y único de cultura que produce exclusión y desigualdad (Aubert, et al., 2010: 88).

Es concluyente que dentro de las construcciones de género existentes, desde el contenido que se enseña la historia de las mujeres, la mujer ha sido silenciada a no ser que alguna actuación femenina coincida con los valores considerados masculinos (García et al., 1993; McIntosh, 2005; Pagès y Sant, 2011, 2012 y Woyshner, 2002).

Por último, y de la misma manera, es concluyente como plantean Croco et al. (1999) y Crocco (2006), Miller (1996) y Schmeichel (2011), que las construcciones y estereotipos de género provocan que la atención prestada 
a las mujeres en la historia sea escasa e inconsistente, en contraste a una historia de los hombres que es presentada como objetiva y relevante. Para Pagès y Sant (2012) es totalmente necesario, si se quiere formar alumnas y alumnos comprometidos con el respeto, la igualdad y la diversidad, cambiar las perspectivas que se están aplicando en las aulas. Desde la organización de los centros, hasta el contenido que se está trabajando. El y la autora proponen que la enseñanza de la historia y las ciencias sociales:

"1. Ejemplifique las formas de dominación mediante ejemplos desde las perspectivas de las mujeres, desde la perspectiva de las clases populares (hombres y mujeres), desde la perspectiva de las minorías étnicas no dominantes (mujeres y hombres, ricos y pobres), desde las perspectivas de los niños (mujeres y hombres, pobres y ricos, negros, asiáticos, blancos, mulatos, etc.) y desde cualquier otra perspectiva de los oprimidos.

2. Seleccione estos ejemplos en base a la proporcionalidad de las personas que existen en el mundo y sin olvidar las minorías. Es decir, no podemos caer en el error de trabajar únicamente la perspectiva de una mujer y la perspectiva de cuatro hombres, o de trabajar la perspectiva de cuatro personas blancas, una asiática y una negra, o de olvidarnos de determinados grupos oprimidos por ser estos una minoría.

3. Utilice ejemplos como fin para mostrar los mecanismos de dominación y opresión y puedan ser extrapolados a otros tipos de dominación ya sea de clase, de género, de etnia, etc.

4. Se base en problemas sociales relevantes puesto que el trabajo por problemas facilita actividades como los estudios de caso o los debates y simulaciones, y se aleja de los contenidos y las estrategias más expositivas que tienden a la socialización y a la reproducción de los valores hegemónicos" (Pagès y Sant, 2012: 116).

En definitiva, y como plantea Subirats (1998), en los tiempos actuales es fundamental que el trabajo de las ciencias sociales y la inclusión de la historia de las mujeres colabore en el fin de los estereotipos y las construcciones androcéntricas del conocimiento y los centros educativos. Depende de los cambios de enfoques y las finalidades que manifestemos al enseñar historia y ciencias sociales, los resultados que obtendremos. La escuela, como afirma Fernández (2004), puede actuar como "potenciador" o "catalizador" de permanencias o cambios en diferentes direcciones. Repensar la enseñanza de la historia y las ciencias sociales es el objetivo primordial. 


\section{Referencias bibliográficas}

Agacinski, S. (1998). Política de sexos. Madrid: Taurus.

Aguirre Sáez de Egilaz, A. (2002). "Orientar para la igualdad, orientar desde la diferencia", en A. González y C. Lomas (coords.), Mujer y Educación. Barcelona: Graó, pp. 183-192.

Álvarez de Zayas, R. M. \& Palomo Alemán, A. (2002). "Los protagonistas de la historia. Los alumnos "descubren" que los hombres comunes también hacen historia", en Enseñanza de las Ciencias Sociales, 1, pp. 27-39.

Apple, M W. \& Beane, J. A. (2012) (Comps.). Escuelas democráticas. Madrid: Morata.

Applebaum, B. (2004). "Social justice education: Moral agency and the subject of resistance", en Educational Theory, 54 (1), pp. 59-72.

Aristóteles (2006). Política. Madrid: Espasa.

Aubert, A., Duque, E., Fisas, M., \& Valls, R. (2010). Dialogar y Transformar: Pedagogía crítica del siglo XXI. Barcelona: Graó.

Banks, J. A. (2004a). "Introduction: Democratic Citizenship Education in a Multicultural Societies", en J. A. Banks, Diversity and Citizenship Education. San Francisco: Jossey-Bass, pp. 3-16.

Banks, J. A. (2006a). Cultural diversity and education: Foundations, curriculum, and teaching (5th ed.). Boston: Allyn and Bacon.

Banks, J. A. (2006b). Race, culture, and education: The selected works of James A. Banks. London \& New York: Routledge.

Banks, J. A. \& Banks, C. A. (2012). Multicultural education: Issues and perspectives (8th ed.). New York: Wiley.

Banks, J. A., Banks, C. A. M., Cortés, C. E., Hahn, C., Merryfield, M., Moodley, K., Osler, A., Murphy-Shigematsu, S., \& Parker, W. C. (2005). Democracy and Diversity: Principles and Concepts for Educating Citizens in a Global Age. Seattle: Center for Multicultural Education, University of Washington.

Barton, K. \& Levstik, L. (2004). Teaching history for the common good. Mahwah, NJ: Erlbaum.

Benavente, J. \& Núñez, A. (1992). "El androcentrismo en la enseñanza de la historia y la geografía (Ed.)", en Del silencio a la palabra. Madrid: Ministerio de Asuntos Sociales, Instituto de la Mujer, pp. 153-176.

Bickmore, K. (1999). "Elementary curriculum about conflict resolution: Can children handle global politics?", en Theory and Research in Social Education, 27(1), pp. 45-69. 
Bickmore, K. (2002). "How Might Social Education Resist Heterosexism? Facing the Impact of Gender and Sexual Ideology on Citizenship", en Theory and Research in Social Education, 30 (2), pp. 198-216.

Bickmore, K. (2008). "Social justice and the social studies", en L. Levstik \& C. Tyson, Handbook of Research in Social Education. New York: Routledge, pp. 155-171.

Blanco, P. (2004). "La perspectiva de género, una necesidad en la construcción de la ciudadanía. Algunas actividades en la formación del profesorado", en M. I. Vera Y D. Pérez (Eds.). Formación de la Ciudadanía: Las TIC y los nuevos problemas. Alicante: Asociación Universitaria del Profesorado de Didáctica de las Ciencias Sociales.

Bonilla, A. y Martínez, I. (1992). "Análisis del currículo oculto de los modelos sexistas", en M. Moreno (Coord.), Del silencio a la palabra. Coeducación y reforma educativa. Madrid: Ministerio de Asuntos Sociales, Instituto de la Mujer, Colección Estudios, p. 60-92.

Casas, M. (1999). "El concepto de diferenciación en la enseñanza de las ciencias sociales", en Íber (6), (21).

Castles, S. (2004). "Migration, citizenship, and education", en J. A. Banks (Ed.), Diversity and citizenship education: Global perspectives. San Francisco: JosseyBass, pp. 17-48.

Connell, R. W. (1993). "Disruptions: Improper masculinities and schooling", en L. Weis y M. Fine (Eds.), Beyond silenced voices. New York: State University of New York, pp. 191-208.

Crocco, M. S. (2002). "Homophobic hallways: Is anyone listening?", en Theory and Research in Social Education, 30(2), pp. 217-233.

Crocco, M. S. (2006). "Gender and social education: What's the problem?", en E. W. Ross (Ed.), The social studies curriculum: Purposes, problems, and possibilities. Albany: State University of New York Press, pp. 171-193.

Crocco, M. S. (2008). "Gender and sexuality in the social studies", en L. Levstik \& C. Tyson, Handbook of Research in Social Studies Education. NY: Routledge, pp. 172-197.

Crocco, M. S. (2010). "Using Literature to Teach about Others, the case of Shabanu", en W. Parker, Social Studies Today: Research and Practice. New York: Routledge Taylor and Francis Group, pp. 175-182.

Crocco, M. S., Munro, P. \& Weiler, K. (1999). Pedagogies of resistance: Women educator activits 1880-1960. New York: Teachers College Press.

Cummins, J. (2001). "Empowering Minority Students: a Framework for Intervention", en Harvard Educational Review, 71 (4), pp. 649-675. 
Dam, G. T. \& Rijkschroeff, R. (1996). "Teaching women's history in secondary education: Constructing gender identity", en Theory and Research in Social Education , 14 (1), pp. 71-89.

Deusdad, B. (2010). "La educación intercultural en las Ciencias Sociales en Cataluña: Nuevas propuestas didácticas", en Íber (64), pp. 111-120.

Fernández Valencia, A. (2004). "Las mujeres como sujeto histórico: género y enseñanza de la historia", en Didáctica de las Ciencias Experimentales y Sociales (18), pp. 5-24.

Fernández Valencia, A. (2006). "La construcción de identidad desde la perspectiva de género", en Íber, 47, 33-44.

GARCÍA, C. \& ROSET, M. (1992). "Sexismo y coeducación en el área de ciencias sociales. (Ed.)", en Del silencio a la palabra. Madrid: Ministerio de Asuntos Sociales, Instituto de la Mujer, pp. 140-152.

García, M., Troiano I Gomà, H., Zaldívar Sancho, M., \& Subirats, M. (1993). El sexismo en los libros de texto: análisis y propuesta de un sistema de indicadores. Bellaterra: Ministerio de Asuntos Sociales, Instituto de la Mujer.

Harris, I. (1996). "From world peace to peace in the hood: Peace education in a postmodern world", en Journal for a Just and Caring Education, 2 (4), pp. 378395.

Hernández, H. \& Metzger, D. (1996). "Issues-Centered Education for LanguageMinority Students", en R. W. Evans \& D. W. Saxe, Handbook on Teaching Social Studies. New York: NCSS, pp. 111-120.

Hubbard, P. (2013). "Kissing is not a universal right: Sexuality, law and the scales of citizenship", en Geoforum, 49, pp. 224-232.

Mcintosh, P. (2005). "Gender Perspectives on Educating for Global Citizenship", en N. Noddings, Educating Citizens for Global Awareness. New York: Teachers College Press, pp. 22-39.

Miller, J. (1996). School for women. London, UK: Virago.

Oesterreich, H. (2002). "'Outing' Social Justice: Transforming Civic Education Within the Challenges of Heteronormativity, Heterosexism, and Homophobia", en Theory and Research in Social Education, 30 (3), pp. 287-301.

Pagés, J. y Sant, E. (2012). "Las mujeres en la enseñanza de la Historia: ¿Hasta cuándo serán invisibles?", en Cad. Pesq. CDhis, 25 (1), pp. 91-117.

Pagés, J. y Sant, E. (2011). "¿Por qué las mujeres son invisibles en la enseñanza de la historia?", en Revista Historia y Memoria, vol. 3, pp. 129-146. 
Pugh, J. L. \& García, J. (1996). "Issues-Centered Education in Multicultural Environments", en R. W. Evans, \& D. W. Saxe, Handbook on Teaching Social Studies. New York: NCSS, pp. 121-129.

Schmeichel, M. (2011). "Feminism, Neoliberalism, and Social Studies", en Theory and Research in Social Education, 39 (1), 6-30.

Scott, J. W. (2008). Género e Historia. México: FCE.

Smith, R. W. (1996). "Schooling and the formation of male students' gender identities", en Theory and Research in Social Education, 24 (1), 54-70.

Stone, L. (1996). "Feminist Political Theory: Contributions to a conception of Citizenship", en Theory and Research in Social Education, 24 (1), pp. 36-53.

Subirats, M. (1998). Educación y Cultura desde la diversidad de géneros. Los valores y la Didáctica de las Ciencias Sociales. Actas del IX Simposium de Didáctica de las Ciencias Sociales.

Subirats, M. (2001). "¿Qué es educar? De la necesidad de reproducción a la necesidad de cambio", en A. Tomé, \& X. Rambla, Contra el sexismo: coeducación y democracia en la escuela. Madrid: Síntesis, pp. 17-21.

Subirats, M. y Brullet, C. (1988). Rosa y Azul. La transmisión de los géneros en el sistema educativo. Madrid: Instituto de la Mujer.

Thornton, S. (2005). "Incorporating internationalism into the social studies curriculum", en N. Noddings (Ed.), Educating citizens for global awareness. New York: Teachers College Press, pp. 81-92.

Tomé, A. y Rambla, X. (ed.) (2001). Contra el sexismo. Coeducación y democracia en la escuela. Madrid: Síntesis. Universidad Autónoma de Barcelona.

Torney-Purta, J., Lehmann, R., Oswald, H. \& Schultz, W. (2001). Citizenship and education in twenty-eight countries: Civic knowledge and engagement at age fourteen. Amsterdam: IEA.

Tyson, C. \& Hinton-Johnson, K. (2003). "Once upon a time: Teaching about women and social justice", en Social Education, 67(1), pp. 54-57.

Vázquez, X. R. (2003). Educación e igualdad de oportunidades entre sexos. Barcelona: ICE-Horsori.

Vega, C. (2002). "La mujer en la historia y la historia de las mujeres", en A. González, \& C. L. (coords.), Mujer y Educación. Barcelona: Graó, pp. 13-20.

Woyshner, C. (2002). "Political History as Women's History: Toward a More Inclusive Curriculum", en Theory and Research in Social Education, 30 (3), pp. 354-380. 